\title{
Uso de substâncias psicoativas entre estudantes da área da saúde da Universidade Federal de Juiz de Fora
}

\author{
Prevalence of Psycotropics Use Among Students \\ of The Health Area of The Federal University of Juiz De Fora
}

\author{
CRISTINA DOS SANTOS OLIVEIRA ${ }^{1}$ \\ LEONARDO CARVALHO DA FONSECA ${ }^{1}$ \\ ALINE LAIGNIER SOARES-YOSHIKAWA ${ }^{2}$ \\ ISABEL CRISTINA GONÇALVES LEITE ${ }^{3}$
}

\begin{abstract}
RESUMO
Objetivo: O objetivo do presente estudo foi analisar o uso de psicotrópicos entre esses estudantes, levando em consideração o sexo, etapa da vida acadêmica e curso frequentado.

Material e Métodos: Foi realizado um estudo transversal, envolvendo uma amostra de 363 alunos do $1^{\circ}$. e $8^{\circ}$. períodos de sete cursos da área da saúde da Universidade Federal de Juiz de Fora.

Resultados: O uso de bebida fermentada foi relatado por $51,52 \%$ dos alunos, de bebida destilada por $36,09 \%$, e de uso do tabaco por 7,99\%. O uso de substâncias como anfetaminas, inalantes, maconha, alucinógenos, sedativos, opioides, barbitúricos e cocaína vieram em seguida. O álcool, tabaco e inalantes figuram entre os que apresentam o maior tempo pregresso de uso: 4,79, 2,89 e 2,87 anos, respectivamente. Com relação ao uso de ambas as bebidas (fermentadas/destiladas), bebidas destiladas exclusivas e tabaco, as mulheres são maiores consumidoras que os homens $(p<0,001$, exceto para o tabaco $p=0,08)$. Os alunos do primeiro período consomem mais anfetaminas, bebidas fermentadas ou ambos, bebidas e tabaco que o oitavo $(p<0,007)$.

Conclusão: Conclui-se que o uso de psicotrópicos entre os acadêmicos aumenta mesmo quando o processo de formação acadêmica está em fase de conclusão, é maior em mulheres e nos curso de Educação Física e Odontologia. Portanto, se deve rever a forma como esse assunto está sendo abordado nas faculdades estudadas.
\end{abstract}

Palavras-chave: Psicotrópicos. Transtornos Relacionados ao Uso de Substâncias. Estudantes de Ciências da Saúde.

\begin{abstract}
Objective: We conducted a cross-sectional study involving a sample of 363 students in the 1st and 8th periods of seven courses in the health of the Federal University of Juiz de Fora.

Material and Methods: Aimed to examine the use of psychotropic drugs among students in training courses in health, taking into account sex, stage of academic life and the course attended.

Results: The use of fermented beverage was reported by $51.52 \%$ of the students and distilled beverages by $36.09 \%$, followed by tobacco with $7.99 \%$ prevalence of use. The use of other substances such as amphetamines, inhalants, marijuana, hallucinogens, sedatives, opiates, barbiturates and cocaine followed. Alcohol, tobacco and inhalants among those with the largest previous time-use: 4.79, 2.89 and 2.87 years respectively. Regarding the use of both beverages (fermented / distilled), exclusive spirits and tobacco, women are bigger consumers than men $(p<0.001$, except for tobacco $p=0.08)$. Students in the first period consume more amphetamines, fermented beverages, beverages and tobacco both the eighth period ( $p<0.007)$. Conclusion: It is concluded that the use of psychotropic drugs among academics increases even when the academic training process is nearing completion, it is higher in women and in Physical Education and Dentistry courses. Therefore, it is necessary to review the way this subject is being studied. Addressed in the colleges studied.
\end{abstract}

Keywords: Psychotropic Drugs. Substance-Related Disorders. Students, Health Occupation.

Farmacêutico(a) pela Universidade Federal de Juiz de Fora.

Doutoranda em Odontologia área de concentração Odontopediatria pela Universidade Federal de Juiz de Fora.

Profa. Adjunta da Faculdade de Medicina da UFJF, Programa de Pós-Graduação em Saúde Coletiva, Núcleo de Assessoria, Treinamentos e Estudos em Saúde. 


\section{INTRODUÇÃO}

Atualmente, pode-se observar que o uso de substâncias psicoativas na sociedade em geral é alarmante, uma vez que causa impacto social, econômico e na própria saúde dos usuários ${ }^{1}$ e os jovens, principalmente, são muito vulneráveis a isso. ${ }^{2}$ Essa população, principalmente os estudantes universitários, pelas próprias adaptações emocionais, objetiva a busca de novas experiências de comportamento, ${ }^{3}$ e pela vulnerabilidade e convivência com muitas dessas substâncias, e também condições de estudos estressantes, se tornaria mais susceptível ao abuso delas. ${ }^{4,5}$

O consumo de substâncias psicoativas entre universitários pode resultar em consequências negativas, decorrentes de seu uso, no percurso acadêmico, podendo influenciar a atuação desses estudantes. ${ }^{6}$ Muitos estudos associam o consumo de tais substâncias a um comportamento de risco, como a condução insegura, ${ }^{7}$ violência, morte prematura, doenças sexualmente transmissíveis, que vão gerar alto custo para o indivíduo e a sociedade. ${ }^{8}$ Esses problemas não só afetam o desenvolvimento físico, emocional e social dos jovens, mas, também, a permanência na universidade e a qualidade de sua formação para a vida profissional. ${ }^{5} \mathrm{O}$ abuso do álcool, mesmo que ocasional, entre universitários tem o potencial de representar um importante problema para a sociedade.

Os universitários dos cursos de saúde merecem um destaque especial quando se trata do uso de psicotrópicos, pois estes serão responsáveis pela educação em saúde da população leiga no futuro, ${ }^{9}$ terão a responsabilidade na identificação e encaminhamento de pacientes com problemas relacionados ao uso de psicotrópicos e ainda também pelo fato de servirem como modelo para os seus pacientes. ${ }^{4}$

O uso de psicotrópicos por esses estudantes se deve à sensação de proteção, dada pelo hipotético conhecimento adquirido na faculdade. ${ }^{10}$ Isso demonstra que, ao contrário do esperado, esses estudantes revelam um desconhecimento quanto ao tema. A literatura destaca que é necessária maior abordagem nos currículos de graduação, sobre o consumo de substâncias psicoativas e seus impactos para o indivíduo, família e sociedade, bem como a criação de programas preventivos específicos para estudantes universitários. .,6 $^{5}$

Diante do exposto, este estudo teve como objetivo analisar o uso de psicotrópicos entre estudantes dos cursos de formação na área de saúde da Universidade Federal de Juiz de Fora levando em consideração o sexo, a etapa da vida acadêmica (início e final do curso) e o curso frequentado para que se conheça um pouco mais dos estudantes e se possa implementar programas de prevenção secundária nessa população como palestras, campanhas e debates acerca desse assunto.

\section{MATERIAL E MÉTODO}

Este foi um estudo epidemiológico do tipo transversal, tendo como população-alvo os estudantes dos cursos da área de saúde da Universidade Federal de Juiz de Fora (UFJF).

A pesquisa foi aprovada pelo Comitê de Ética e Pesquisa com Seres Humanos da UFJF (protocolo $n^{\circ}$. 309/2007) e os respondentes assinaram o termo de consentimento informado, de acordo com a Resolução $n^{\circ}$. 466/12 do Conselho Nacional de Saúde, que dispõe sobre a pesquisa envolvendo seres humanos. ${ }^{11}$

Segundo a UFJF, fazem parte dos cursos da área de saúde: Ciências Biológicas, Educação Física, Enfermagem, Farmácia e Bioquímica; Odontologia; Fisioterapia e Medicina. A amostra foi distribuída da seguinte forma: 80 estudantes de cada um dos seguintes cursos; Ciências Biológicas, Educação Física, Enfermagem, Farmácia e Bioquímica e Odontologia; 40 alunos do curso 
de Fisioterapia e 160 do curso de Medicina. ${ }^{12}$ O número amostral de entrevistados de cada curso foi dividido em duas partes iguais, sendo uma composta por alunos do primeiro período e a outra por alunos do último período de faculdade com carga teórica no campus universitário.

Os critérios de elegibilidade adotados foram idade superior a 18 anos e todos os alunos regularmente matriculados num desses cursos. O estudo foi conduzido por censo, já que todos os 300 alunos do primeiro período e 300 do último período, com carga teórica no campus universitário de cada curso selecionado, foram convidados a participar. Foi padronizado o oitavo período como sendo o último período de faculdade, com exceção dos cursos de Odontologia e Medicina, isso foi adotado porque, quando esse não era o último período de faculdade, era o último período em que a maioria dos alunos da maior parte dos cursos possuía aula teórica no campus universitário.

Em cada curso e período, escolheu-se a disciplina de maior frequência. Após a escolha da disciplina, fez-se um prévio agendamento da visita dos entrevistadores e, após aquiescência do professor, foi apresentada a proposta do estudo e aplicado o instrumento selecionado aos alunos presentes na sala de aula no dia da aplicação. O questionário foi aplicado para cada curso e período em dias diferentes. A devolução foi feita ao final da aula. A coleta de dados foi realizada pelos próprios autores do estudo, devidamente calibrados por meio de estudo piloto, para destacar a importância do estudo, explicar como o questionário devia ser preenchido, apesar de ele ser autopreenchível e esclarecer qualquer dúvida.

Entretanto, dos 600 alunos matriculados nos sete cursos da área de saúde da Universidade Federal de Juiz de fora, 185 $(30,8 \%)$ não estavam presentes em sala de aula no momento da aplicação do questionário ou, mesmo presentes, se recusaram a participar da pesquisa e $52(8,7 \%)$ alunos entregaram o questionário incompleto. Sendo assim, a amostra final foi constituída de 363 estudantes, desprezando-se para fins estatísticos, as perdas.

O questionário utilizado como instrumento da pesquisa foi elaborado pela World Health Organization (WHO), e adaptado, pelos pesquisadores, para uma versão em português: "WHO opioid evaluation instrument". ${ }^{13} \mathrm{O}$ instrumento era autoexplicativo e garante o anonimato, composto por 27 questões, e dividido em quatro partes. A primeira parte explicava o estudo e convidava os estudantes para dele participar (termo de consentimento livre e esclarecido - TCLE). A segunda parte era sobre variáveis classificatórias (sexo, curso, período de faculdade e local de residência de seus responsáveis). $\mathrm{Na}$ terceira, havia a pesquisa a respeito do uso de drogas, que questionava o sujeito da pesquisa acerca do uso de bebida alcoólica destilada e fermentada, tabaco, heroína, outros opioides/ analgésicos, barbitúricos, outros sedativos e tranquilizantes, cocaína e seus derivados, anfetaminas, maconha, alucinógenos e inalantes.

Os dados foram armazenados no programa EPI INFO 3.3.2. A análise dos dados baseouse no teste $t$ de Student para comparação de médias e $x^{2}$ para comparação de proporções. Foi admitido erro alfa de $5 \%$.

\section{Resultados}

Participaram deste estudo 363 universitários. A maioria dos participantes era do sexo feminino (71\%), não havendo diferença significativa com relação à distribuição da população-alvo. Não houve uma diferença significante quanto ao período de faculdade cursado, sendo que $58 \%$ dos entrevistados cursavam o primeiro período, e quanto ao local 
de procedência do aluno (moradia da família) 52,7\% afirmaram que a família morava no município de Juiz de Fora. O curso de Medicina foi o curso que teve um maior número de participantes (22,6\% do total da amostra), sendo também que este é o curso que possui maior número de alunos matriculados (Tabela 1).

Tabela 1 - Distribuição dos estudantes segundo as características sociodemográficas.

\begin{tabular}{|c|c|c|c|c|c|c|c|c|}
\hline Curso & $\begin{array}{l}\text { Cienc } \\
\text { Biol }\end{array}$ & Ed. Fis & Enf. & Farm. & Fisiot. & Med. & Odont. & $\begin{array}{l}\text { Total de } \\
\text { respostas } \\
\text { obtidas }\end{array}$ \\
\hline $\begin{array}{l}\boldsymbol{n} \text { ( cada } \\
\text { subamostra) }\end{array}$ & $\begin{array}{c}35 \\
(9,6 \%)\end{array}$ & $\begin{array}{c}43 \\
(11,9 \%)\end{array}$ & $\begin{array}{c}53 \\
(14,6 \%)\end{array}$ & $\begin{array}{c}55 \\
(15,2 \%)\end{array}$ & $\begin{array}{c}34 \\
(9,4 \%)\end{array}$ & $\begin{array}{c}82 \\
(22,6 \%)\end{array}$ & $\begin{array}{c}61 \\
(16,8 \%)\end{array}$ & $\begin{array}{c}363 \\
(100 \%)\end{array}$ \\
\hline $\begin{array}{l}\text { Origem } \\
\text { Juiz de Fora } \\
\text { Outra cidade }\end{array}$ & $\begin{array}{c}17 \\
(9,2 \%)\end{array}$ & $\begin{array}{c}31 \\
(16,7 \%)\end{array}$ & $\begin{array}{c}26 \\
(14,1 \%)\end{array}$ & $\begin{array}{c}31 \\
(16,7 \%)\end{array}$ & $\begin{array}{c}17 \\
(9,2 \%)\end{array}$ & $\begin{array}{c}41 \\
(22,2 \%)\end{array}$ & $\begin{array}{c}22 \\
(11,9 \%)\end{array}$ & $\begin{array}{c}185 \\
(52,7 \%)\end{array}$ \\
\hline \multirow[t]{2}{*}{$p$} & $\begin{array}{c}16 \\
(9,6 \%)\end{array}$ & $\begin{array}{c}12 \\
(7,2 \%)\end{array}$ & $\begin{array}{c}23 \\
(13,9 \%)\end{array}$ & $\begin{array}{c}22 \\
(13,2 \%)\end{array}$ & $\begin{array}{c}17 \\
(10,2 \%)\end{array}$ & $\begin{array}{c}41 \\
(24,7 \%)\end{array}$ & $\begin{array}{c}35 \\
(21,2 \%)\end{array}$ & $\begin{array}{c}166 \\
(47,4 \%)\end{array}$ \\
\hline & 0,67 & $<0,01$ & 0,98 & 0,59 & 0,67 & 0,71 & $<0,03$ & 0,16 \\
\hline $\begin{array}{l}\text { Período: } \\
1^{\circ} \text {. período }\end{array}$ & $\begin{array}{c}22 \\
(10,4 \%)\end{array}$ & $\begin{array}{c}14 \\
(6.6 \%)\end{array}$ & $\begin{array}{c}39 \\
(18,5 \%)\end{array}$ & $\begin{array}{c}37 \\
(17,6)\end{array}$ & $\begin{array}{c}21 \\
(10 \%)\end{array}$ & $\begin{array}{c}49 \\
(23,2 \%)\end{array}$ & $\begin{array}{c}29 \\
(13,7 \%)\end{array}$ & $\begin{array}{c}211 \\
(58 \%)\end{array}$ \\
\hline \multicolumn{9}{|l|}{ Último período } \\
\hline \multirow{2}{*}{$p$} & $\begin{array}{c}13 \\
(8,5 \%)\end{array}$ & $\begin{array}{c}29 \\
(19,1 \%)\end{array}$ & $\begin{array}{c}14 \\
(9,2 \%)\end{array}$ & $\begin{array}{c}18 \\
(11,9 \%)\end{array}$ & $\begin{array}{c}13 \\
(8,6 \%)\end{array}$ & $\begin{array}{c}33 \\
(21,7 \%)\end{array}$ & $\begin{array}{c}32 \\
(21 \%)\end{array}$ & $\begin{array}{c}152 \\
(42 \%)\end{array}$ \\
\hline & 0,98 & $<0,01$ & 0,16 & 0,43 & 0,67 & 0,85 & 0,05 & $<0,01$ \\
\hline $\begin{array}{l}\text { Sexo: } \\
\text { Masculino }\end{array}$ & $\begin{array}{c}6 \\
(5,8 \%)\end{array}$ & $\begin{array}{c}21 \\
(20,2 \%)\end{array}$ & $\begin{array}{c}4 \\
(3,9 \%)\end{array}$ & $\begin{array}{c}20 \\
(19,2 \%)\end{array}$ & $\begin{array}{c}7 \\
(6,7 \%)\end{array}$ & $\begin{array}{c}28 \\
(26,9 \%)\end{array}$ & $\begin{array}{c}18 \\
(17,3 \%)\end{array}$ & $\begin{array}{c}104 \\
(29 \%)\end{array}$ \\
\hline Feminino & $\begin{array}{c}29 \\
(11,3 \%)\end{array}$ & $\begin{array}{c}22 \\
(8,6 \%)\end{array}$ & $\begin{array}{c}49 \\
(19,1 \%)\end{array}$ & $\begin{array}{c}35 \\
(13,6 \%)\end{array}$ & $\begin{array}{c}27 \\
(10,5 \%)\end{array}$ & $\begin{array}{c}53 \\
(20,6 \%)\end{array}$ & $\begin{array}{c}42 \\
(16,3 \%)\end{array}$ & $\begin{array}{l}257 \\
(71 \%)\end{array}$ \\
\hline$p$ & 0,67 & 0,13 & $<0,01$ & 0,30 & 0,67 & 0,36 & 0,81 & $<0,01$ \\
\hline
\end{tabular}

Legenda: Cienc Biol.: Ciências Biológicas, Educ Fis: Educação Física, Enf: Enfermagem, Farm: Farmácia, Fisiot; Fisioterapia, Med: Medicina, Odont: Odontologia, "n": Número.

p- Teste de Student- a $5 \%$ 
A análise dos dados citada permite observar que há homogeneidade entre as variáveis analisadas. Contudo, em relação à origem da família dos entrevistados, no curso de Educação Física houve uma diferença significativa quando comparados os alunos de Juiz de Fora com alunos vindos de outras cidades $(p=0,006)$. A diferença também é vista no curso de Odontologia $(p=0,021)$, porém, nesse curso, ao contrário do que ocorre no curso de Educação Física, o número de estudantes de outra cidade é maior. Esses dois cursos também mostram comportamentos diferentes em relação à participação dos alunos quanto ao período, uma vez que a participação do número de estudantes do último período foi maior que no primeiro período, para os cursos de Educação Física $(p<0,001)$ e Odontologia $(p=0,053)$. Ao avaliar a distribuição por sexo, apenas o curso de Enfermagem apresenta diferença significativa $(p<0,001)$.

$\mathrm{Na}$ Tabela 2 podem ser observados dados sobre o uso de drogas psicotrópicas nos últimos 30 dias, nela se percebe que as drogas lícitas foram as mais utilizadas. Entre elas, as bebidas fermentadas foram as mais relatadas pelos estudantes $(51,52 \%)$, seguidas das bebidas destiladas $(36,09 \%)$, sendo que $25,62 \%$ fizeram uso de ambas as bebidas (destilada/fermentada). O consumo de tabaco foi relatado por $7,99 \%$ da amostra.

Tabela 2 - Prevalência do uso de drogas psicotrópicas nos últimos 30 dias entre os estudantes.

\begin{tabular}{ccc}
\hline Droga & Frequência absoluta & Frequência relativa \\
\hline Beb. Fermentadas & 187 & 51,52 \\
Beb. Destiladas & 131 & 36,09 \\
Beb. Ambas & 93 & 25,62 \\
Tabaco & 29 & 7,99 \\
Inalante & 8 & 2,20 \\
Anfetamina & 8 & 2,20 \\
Maconha & 6 & 1,65 \\
Opioides & 3 & 0,83 \\
Sedativos & 3 & 0,83 \\
Alucinógeno & 3 & 0,83 \\
Barbitúricos & 1 & 0,28 \\
Cocaína & 1 & 0,28 \\
\hline
\end{tabular}

Legenda: Beb. Fermentadas: Bebidas Fermentadas, Beb. Destiladas: Bebidas Destiladas, Beb. Ambas: Bebidas Ambas (tanto destiladas como fermentadas).

Com relação às drogas ilícitas, as mais usadas nos últimos 30 dias foram as anfetaminas e os inalantes $(2,20 \%$ cada), seguidas pela maconha com $1,65 \%$ e pelos alucinógenos, opioides e sedativos $(0,83 \%$ cada). Os barbitúricos e a cocaína foram as drogas menos usadas pelos alunos $(0,28 \%$ cada).

Juntamente ao questionário que avaliava o uso pregresso de drogas nos últimos 30 dias havia a opção de marcação se o mesmo não fazia uso de qualquer droga. Com isso, foi possível observar que muitos deles assumem o uso de álcool ou tabaco, mas não consideram que usam drogas, ou seja, não consideram álcool ou tabaco como sendo uma droga. Entre os 187 alunos que afirmaram usar bebidas fermentadas, $70,05 \%$ não consideram esse tipo de bebida como droga. Em relação às bebidas destiladas, 47,33\% dos usuários fizeram essa mesma afirmação. Dos que usam ambas as bebidas, esse valor é de 44,09\%. Quanto ao tabaco, o percentual de estudantes tabagistas 
que negam usar drogas é de 27,6\%. Porém, $3,97 \%$ dos que asseveraram que faziam uso de drogas, usavam álcool (fermentado, destilado ou ambos), em um mesmo intervalo de tempo, com outras drogas; $5,47 \%$ usavam tabaco com outras drogas e ainda 1,55\% usavam álcool, tabaco e outras drogas concomitantemente.

A Tabela 3 mostra a distribuição do uso das drogas segundo o sexo. Houve um predomínio do uso de drogas psicotrópicas legais entre as mulheres e o uso de drogas psicotrópicas ilegais entre os homens. Porém, diferenças estatisticamente significativas com relação ao sexo foram encontradas para as bebidas fermentadas $(p=0,002)$, ambas as bebidas e tabaco $(p=0,054)$. Bebidas destiladas apresentam valores marginalmente significativos $(p=0,073)$.

Com relação ao período de faculdade, observamos que o uso de drogas psicotrópicas legais foi maior no primeiro período e o uso de drogas psicotrópicas ilegais no oitavo período. A frequência do uso de sedativos foi a mesmo para ambos os períodos de faculdade (Tabela 3).

Tabela 3 - Relato sobre uso de drogas psicotrópicas usadas pelos estudantes segundo sexo, período cursado na faculdade, local de moradia dos pais ou responsáveis e tempo médio de uso.

\begin{tabular}{|c|c|c|c|c|c|c|c|c|c|c|c|}
\hline $\begin{array}{l}\text { Droga } \\
\text { Variável }\end{array}$ & Aluc. & Anfet. & $\begin{array}{c}\text { Bar } \\
\text { bit }\end{array}$ & Dest & Ferm & $\begin{array}{l}\text { Beb } \\
\text { Geral }\end{array}$ & Inal. & $\begin{array}{c}\text { Mac } \\
.\end{array}$ & Opio. & $\begin{array}{c}\text { Sedat } \\
.\end{array}$ & Tab. \\
\hline \multicolumn{12}{|l|}{ Sexo } \\
\hline Fem. & - & 3 & 1 & 86 & 16 & 59 & 5 & 1 & 1 & 1 & 16 \\
\hline Masc. & 3 & 5 & - & 45 & 171 & 34 & 3 & 6 & 2 & 3 & 13 \\
\hline Período & & & & & & & & & & & \\
\hline $\begin{array}{c}1^{\circ} . \\
\text { período }\end{array}$ & - & 5 & - & 42 & 131 & 53 & 2 & 3 & 3 & 2 & 24 \\
\hline $\begin{array}{l}\text { Outro } \\
\text { período }\end{array}$ & 3 & 3 & 1 & 89 & 56 & 40 & 6 & 4 & - & 2 & 5 \\
\hline Origem & & & & & & & & & & & \\
\hline $\begin{array}{c}\text { Juiz de } \\
\text { Fora }\end{array}$ & 1 & 3 & 1 & 87 & 64 & 43 & 5 & 5 & 2 & - & 9 \\
\hline $\begin{array}{l}\text { Outra } \\
\text { cidade }\end{array}$ & 2 & 5 & - & 44 & 123 & 50 & 3 & 2 & 1 & 4 & 20 \\
\hline $\begin{array}{l}\text { Tempo } \\
\text { médio } \\
\text { de uso } \\
\text { (anos) }\end{array}$ & 1,67 & 2 & 6 & 4,5 & 5 & 4,79 & 2,87 & 2,57 & 5 & 2,75 & $\begin{array}{c}2,8 \\
9\end{array}$ \\
\hline
\end{tabular}

Legenda: Aluc.: Alucinógenos, Anfet: Anfetamina, Barbit.: Barbitúrico, Dest: Bebida Destilada, Ferm: Bebida Fermentada, Beb geral: Ambas as Bebidas, Inal.: Inalantes, Mac. Maconha, Opio. Opioides, Sedat.: Sedativos, Tab.: Tabaco. 
Não houve diferença quanto ao uso da maioria das drogas psicotrópicas ilícitas (exceto alucinógenos e anfetaminas) entre a presença ou ausência da família na cidade, mas o predomínio foi entre os alunos cujos pais moram em outra cidade (Tabela 3 ).

Analisando o tempo médio de uso (em anos) das drogas pesquisadas, excetuando-se barbitúricos e opioides, que foram relatados por apenas um e dois estudantes respectivamente, o álcool $(4,79)$ e o tabaco $(2,89)$ foram as drogas que apresentaram maiores médias de uso pregresso e as menores foram os alucinógenos $(1,67)$ e anfetaminas $(2,0)$. O tempo de uso em média das drogas variou de 1,67 a 6,0 anos (Tabela 3).

A Tabela 4 mostra a escala de dependência dos participantes em estudo. Nela podemos notar que houve predomínio de uma resposta para todas as perguntas avaliadas, pois a maioria dos sujeitos da pesquisa respondeu o item que recebia pontuação zero (0) (máximo - $97,8 \%$ e mínimo - 80,4\%). Todas as questões e todos os outros números também foram pontuados, porém com prevalência bem menor.

Tabela 4 - Distribuição da escala de dependência do uso de drogas usadas pelos estudantes, segundo a pontuação.

\begin{tabular}{|c|c|c|c|c|}
\hline Variável & $\begin{array}{c}\text { Nuncal } \\
\text { Quase nunca } \\
0\end{array}$ & $\begin{array}{l}\text { Algumas } \\
\text { Vezes } \\
1\end{array}$ & $\begin{array}{c}\text { Frequentemente } \\
2\end{array}$ & $\begin{array}{c}\text { Sempre/ } \\
\text { Quase } \\
\text { sempre } \\
3\end{array}$ \\
\hline $\begin{array}{c}\text { Quanto ao seu uso de drogas } \\
\text { é fora de controle } \\
\text { Frequência }\end{array}$ & 206 & 15 & 2 & 1 \\
\hline (\%) & 92,0 & 6,7 & 0,9 & 0,4 \\
\hline
\end{tabular}

Quanto à possibilidade da falta de consumir uma dose o deixa ansioso ou preocupado $219 \quad 5$ Frequência

Prevalência (\%) 97,8 2,2

\begin{tabular}{c} 
Prevalência (\%) \\
\hline Quanto se Preocupa com seu \\
uso de droga \\
Frequência
\end{tabular}

Prevalência (\%) 192 22 5 5

\begin{tabular}{|c|c|c|c|c|}
\hline Prevalência (\%) & 85,7 & 9,8 & 2,2 & 2,2 \\
\hline $\begin{array}{c}\text { Quanto se gostaria de parar } \\
\text { de usar drogas } \\
\text { Frequência }\end{array}$ & 180 & 30 & 7 & 7 \\
\hline \multirow[t]{2}{*}{ Prevalência (\%) } & 80,4 & 13,4 & 3,1 & 3,1 \\
\hline & $\begin{array}{c}\text { Nenhuma } \\
\text { dificuldade } \\
0\end{array}$ & $\begin{array}{c}\text { Pouca } \\
\text { dificuldade } \\
1\end{array}$ & $\begin{array}{l}\text { Muita dificuldade } \\
2\end{array}$ & $\begin{array}{c}\text { Impossível } \\
3\end{array}$ \\
\hline $\begin{array}{c}\text { Quanto à dificuldade de ficar } \\
\text { sem usar droga } \\
\text { Frequência }\end{array}$ & 200 & 22 & 2 & - \\
\hline Prevalência (\%) & 89,3 & 9,8 & 0,9 & - \\
\hline
\end{tabular}




\section{DIscussão}

Os resultados encontrados informam que a amostra deste estudo é composta por uma grande parte de mulheres. Isso pode ser justificado pelo fato de que os cursos de saúde são compostos na maioria por mulheres, o mesmo foi encontrado por Nunes et al. $(2012)^{9}$ realizado em Minas Gerais, com $62,4 \%{ }^{15} \quad$ Uma limitação deste estudo foi a perda amostral, em que do total de 600 estudantes elegíveis, 52 (8,7\%) deixaram o questionário incompleto, e 363 aceitaram participar da pesquisa. A pesquisa corrobora com outros estudos em relação ao uso de álcool e tabaco ${ }^{16}$. Neste artigo, encontrouse que mais da metade dos entrevistados fazem uso de álcool e 7,99 \% usam tabaco. Em estudo feito por Eckschmidt, Andrade, Oliveira (2013), ${ }^{1}$ realizado na Universidade de São Paulo, eles encontraram uma prevalência de consumo de álcool $89,3 \%$ e de $45,5 \%$ para o consumo de tabaco entre os estudantes de medicina. Neste estudo, o álcool foi a droga mais consumida. Segundo o Ministério da Saúde (MS, 2020), ${ }^{15}$, é uma droga de consumo admitido e incentivado nas relações sociais. Castaño-Perez e CalderonVallejo (2014), ${ }^{5}$ em estudo realizado com universitários, encontraram que $88,6 \%$ da amostra fazia consumo de álcool e no estudo de Nunes et al. (2012), feito com universitários de Minas Gerais, a prevalência foi de $71,5 \%{ }^{9}$

$O$ uso de bebida destilada entre as mulheres supera o uso por homens (86 e 45 respectivamente), bem como, quando analisado o uso de ambas as bebidas (59 e 34 respectivamente) e o uso de tabaco (16 e 13 respectivamente). Em relação ao álcool, este achado é concordante com o de Lucas et al. (2006). ${ }^{4}$ Os estudantes do primeiro período apresentam menor uso de bebidas destiladas (7\%) que os do último período $(13,3 \%)$, porém em relação ao tabaco, o consumo pelos estudantes do primeiro período é bem maior (4\%), ${ }^{17}$ o primeiro dado mostra que o conhecimento adquirido na faculdade não está sendo aplicado na busca de uma vida saudável, enquanto que o segundo confirma a necessidade de intervenção no sentido de prevenção no início do curso, sugerido por Almeida et al. $(2011)^{16}$ em um estudo realizado em Lins. Os autores observaram que a maioria dos universitários $(80 \%)$ iniciava o uso de tabaco no primeiro período. ${ }^{17}$

De acordo com o Instituto Nacional de Câncer, ${ }^{18}$ o tabaco é a segunda droga mais consumida entre os jovens no Brasil e no mundo, o que se deve às facilidades e estímulos para a obtenção do produto. Além disso, 90\% dos fumantes iniciaram seu uso antes dos 19 anos de idade. Para diminuir o uso de tabaco no Brasil o INCA coordena o Programa de Combate ao Tabaco e Outros Fatores de Risco de Câncer que se baseia na adoção de medidas que visem um comportamento e estilo de vida saudável, já que o tabaco é considerado o principal causador de mortes evitáveis.

Dados do INCA ainda apontam que $7,1 \%$ dos jovens, entre 15 e 24 anos de idade, da cidade do Rio de Janeiro, $11,7 \%$ de Belo Horizonte, e $24,1 \%$ de Porto Alegre são fumantes, o que vem reforçar os dados referentes ao uso dessas drogas em idade precoce. E ainda, nos países desenvolvidos os fumantes constituem $48 \%$ da população masculina e $7 \%$ da população feminina. Caso as atuais tendências de expansão do consumo de tabaco sejam mantidas, o número de mortes anuais irá para 10 milhões por volta do ano 2030, sendo metade delas em indivíduos jovens (até 35 anos de idade). ${ }^{18}$ Almeida et al. $(2011)^{16}$ ao compararem a prevalência de tabagismo encontrada ao pesquisar universitários da região de Lins $(11,7 \%)$, com prevalência de estudos anteriores, concluíram que as prevalências são semelhantes, podendo demonstrar que as medidas antitabágicas já 
existentes não estão sendo eficazes ou estão deixando de ser implementadas.

Os estudantes cujos pais vivem em outras cidades apresentam um consumo de bebidas fermentada e tabaco significativamente maior que os estudantes cuja origem é Juiz de Fora. ${ }^{19,20,21}$ Uma possível explicação decorre do fato de que os pais dos estudantes de outras cidades não têm participação tão ativa na vida dos filhos quanto os pais que moram com os filhos em Juiz de Fora, ou então porque usam a bebida e o cigarro como fuga da solidão e saudade da família e também para sociabilização. ${ }^{21}$

Outras drogas citadas foram anfetaminas e inalantes, maconha, alucinógenos, opioides e sedativos, barbitúricos e cocaína. Uma única pessoa relatou fazer uso de barbitúricos e o faz há seis anos durante 30 dias por mês. $O$ caso se trata de um uso crônico, e sob a supervisão de um médico, e também dois universitários relataram que utilizavam opioides, em média, por cinco anos. E quanto a uma possível interferência de sexo, os homens universitários usam maconha com mais frequência que as universitárias da mesma faixa etária $(34,5 \%$ vs. $21,4 \%)$.

Considerando o uso ao longo da vida, o álcool é potencialmente a primeira droga a ser experimentada, chegando, em alguns casos, a ter uso superior a seis anos, independentemente da natureza da bebida. Essa precocidade também ocorre com o tabaco (média de uso 2,89 anos) e a maconha (média de uso 2,57 anos) de o uso de a maconha relacionar-se com o uso posterior de outras drogas.

Essa pesquisa revelou também que $66,67 \%$ dos sujeitos faziam uso somente de álcool e/ou tabaco, e/ou ambos relataram que não faziam uso de qualquer tipo de droga, isso é um dado preocupante, pois, pode ser um indicativo de que muitos estudantes na área de saúde ainda não têm o conhecimento completo do conceito de droga porque não consideram que álcool e tabaco são tipos de drogas. Esses resultados sugerem que o modo como o assunto é abordado nas faculdades e em salas de aula deveria ser revisto, pois o consumo de substâncias psicoativas ainda é alto entre esses estudantes.

O conhecimento das particularidades do uso de substâncias psicoativas entre os universitários possui grande importância para a detecção precoce desse consumo. Investimentos nesse segmento devem ser contemplados nas políticas preventivas uma vez que possuem a melhor relação custobenefício. ${ }^{14,16}$

\section{CONSIDERAÇÕES FINAIS}

De maneira geral, a prevalência do uso de psicotrópicos entre acadêmicos dos cursos da área da saúde da Universidade Federal de Juiz de Fora apresentou diferenças quantitativas entre aqueles do início e do final do curso, quando foram comparados estudantes no início do curso de graduação e na iminência de sua formação podendo ser reflexo dos conhecimentos adquiridos durante a graduação. Entre os cursos, houve incremento no uso nos cursos de Educação Física e Odontologia com o avanço da formação universitária. Com relação ao tipo de droga, observou-se particularmente o uso da bebida destilada, porém não há explicações exatas para a ocorrência de tal fato.

O presente estudo permitiu fornecer subsídios às futuras políticas institucionais de prevenção ao uso de substâncias psicotrópicas voltadas para o meio universitário de forma adequada, visto que o uso dessas substâncias persiste mesmo com sua formação estar na iminência de conclusão. Esse é um problema detectado em outros estudos nacionais, constituindo-se, portanto, em importante objeto de intervenção. 


\section{REFERÊNCIAS}

1. Souza J., Ornella KP, Almeida LY, Domingos SGA, Andrade LS, Zanetti ACG. Drug use and knowledge of its consequences among nursing students. enferm. [online], 2018, v. 27, n. 2, e 5540016. https://doi. org/10.1590/0104-070720180005540016.

2. Midford R., Ramsden R., Lester L., Cahill H., Mitchell J., Foxcroft DR, Venning L. Alcohol Prevention and School Students: Findings From an Australian 2-Year Trial of Integrated Harm Minimization School Drug Education. J Drug Educ. 2015; 0 (0): 1-24. doi: 10.1177/0047237915579886.

3. Pillon SC, O'brien B, Chavez KAP. The relationship between drugs use and risk behaviors in brazilian university students. Revista Latino-Americana de Enfermagem. 2005; 13 (especial). https://doi.org/10.1590/ S0104-11692005000800011

4. Lucas ACSL, Parente RCPP, Picanço NS, Conceição DA, Costa KRC, Magalhães IRS, Siquera JCA. Uso de psicotrópicos entre universitários da área da saúde da Universidade Federal do Amazonas, Brasil. Caderno de Saúde Pública, 2006; 22 (3): 673-71. https:// doi.org/10.1590/S0102-311X2006000300021.

5. Castaño-Perez GA, Calderon-Valejjo GA. Problems associated with alcohol consumption by university students. Rev Lat Am Enfermagem. 2014, Sep./Oct.; 22 (5): 739-746. doi: 10.1590/0104-1169.3579.2475

6. Santos, MVF, Ferreira DS, Siqueira MM. Uso de álcool e tabaco entre estudantes de Psicologia da Universidade Federal do Espírito Santo. J Bras Psiquiatr. 2013; 62 (1): 22-30. https://doi. org/10.1590/S0047-20852013000100004.

7. Martz ME, Patrick ME, Schulenberg JE. Alcohol mixed with energy drink use among u.s. 12thgrade students: prevalence, correlates, and associations with unsafe driving. J Adolesc Health. 2015; 56 (5): 557-63. doi: 10.1016/j. jadohealth.2015.01.019.

8. Oliveira EB, Cunningham J., Strike C., Brands B., Wright MGM. Normas percebidas por estudantes universitários sobre o uso de álcool pelos pares. Rev Latino-Am Enfermagem 2009; 17 (Esp.): 878-85. https://doi.org/10.1590/ S0104-11692009000700019

9. Nunes JM, Campolina LR, Vieira MA, Caldeira AP. Consumo de bebidas alcoólicas e prática do binge drinking entre acadêmicos da área da saúde. Rev Psiq Clín. 2012; 39 (3): 94-9. https:// doi.org/10.1590/S0101-60832012000300005.

10. Kerr-Corrêa F., Andrade AG, Bassit AZ, Boccuto NMVF. Uso de álcool e drogas por estudantes de medicina da Unesp. Rev Bras Psiquiatr, 1999; 21 (2): 95-100. https://doi.org/10.1590/ S1516-44461999000200005.

11. BRASIL. Ministério da Saúde. Conselho Nacional de Saúde. Resoluçãon n . 196.10/10/96. Diretrizes e Normas Regulamentadoras de Pesquisa em Seres Humanos. Brasília, DF, 1996. https://doi.org/10.1590/S151774912003000500006

12. UNIVERSIDADE FEDERAL DE JUIZ DE FORA - UFJF. Cursos da área de Saúde. Disponível em: <http://www.ufjf.br/prograd>. Acesso em: 17 fev. 2020.

13. WORLD HEALTH ORGANIZATION - WHO. Instrumento utilizado na pequisa. (WHO opioid evaluaton instrument). Disponível em: http://www.who.int/substance_abuse/research_ tools/en/english_asi.pdf. 17 fev. 2020.

14. Eckschmidt F., Andrade AG, Oliveira LG. Comparação do uso de drogas entre universitários brasileiros, norte-americanos e jovens da população geral brasileira. J Bras Psiquiatr. 2013; 62 (3): 199-207. https://doi. org/10.1590/S0047-20852013000300004

15. MS - MINISTÉRIO DA SAÚDE. Alcoolismo. Disponível em: <https://www.saude.gov.br/ saude-para-voce/saude-do-adolescente-e-dojovem/uso-de-alcool-e-outras-drogas>. Acesso em: 7 fev. 2020.

16. Almeida JB, Miranda JS, Miyasaki SCS, Marques SFG. Prevalência e características do tabagismo na população universitária da região de Lins-SP. Rev Bras Enferm. 2011; 64 (3): 545-50. https://doi.org/10.1590/S003471672011000300019.

17. Franco C., Colares V. Estudo comparativo de condutas de saúde entre universitários no início 
e no final do curso. Revista de Saúde Pública. 2008; 42 (3): 420- 427. https://doi.org/10.1590/ S0034-89102008000300005.

18. INCA - Instituto Nacional do Câncer. Estatísticas sobre tabagismo. Disponível em: http://www.inca.gov.br/tabagismo. Acesso em: 7 fev. 2020.

19. Peuker AC, Fogaça J., Bizarro L. Expectativas e Beber Problemático entre Universitários. Psicologia: Teoria e Pesquisa. 2006; 22 (2): 193-200. https://doi.org/10.1590/S010237722006000200009 .
20. Silva EC, Tucci AM. Estudo transversal sobre o uso de risco de álcool em uma amostra de estudantes de uma universidade federal brasileira. J Bras Psiquiatr. 2014; 63 (4): 317-25. https://doi.org/10.1590/0047-2085000000040.

21. Vieira PC, Aerts DRGC, Freddo SL, Bittencourt A., Monteiro L. Uso de álcool, tabaco e outras drogas por adolescentes escolares em município do Sul do Brasil. Cadernos de Saúde Pública. 2008; 24 (11): 2.487-2.498. https://doi. org/10.1590/S0102-311X2008001100004.

Submetido em: 26-11-2018

Aceito em: 18-2-2020 